\title{
THE POPULATION GENETICS OF SEX DETERMINATION IN HONEY BEES: RANDOM MATING IN CLOSED POPULATIONS
}

\author{
ROBERT E. PAGE, JR.* AND R. WILLIAM MARKS + \\ * Department of Entomology, University of California, Davis, California 95616, U.S.A. \\ + Museum of Comparative Zoology, Harvard University, Cambridge, Massachusetts \\ 02138, U.S.A.
}

Received 4.ix.81

\section{SUMMARY}

\begin{abstract}
The genetic load associated with the method of sex determination in honey bees has been a great deterrent to selective breeding programmes, primarily because of a lack of understanding of the population genetics of the system. In this paper we examine the distribution of diploid brood viabilities in closed, random mating populations, as a function both of the system of sex determination and of multiple mating in queens. Analytically we show that for a given number of sex alleles, an increase in the numbers of matings of queens, reduces the variance in brood viability, but does not affect the mean. The results of a computer simulation demonstrate that the equilibrium number of sex alleles is approached very slowly from above, so that small populations that start with large numbers of sex alleles will maintain high brood viability for a long time. The practical significance of this is discussed.
\end{abstract}

\section{INTRODUCTION}

SEX in honey bees is determined at a single locus with multiple alleles (Mackensen, 1951). Females are heterozygous at this locus. Homozygotes are diploid males; normal males are haploid. Larval diploid males are detected by the workers in the colony soon after eclosion from the egg and are consumed (Woyke 1963) making homozygosity at this locus effectively lethal.

With instrumental insemination it is possible to establish breeding populations that are completely closed to gene flow from surrounding natural populations. These closed populations could be important to honey bee stock maintenance and selection programmes, especially in areas threatened by introgression of genes from "Africanized" bee populations. However, the genetic load associated with sex determination, severe loss of colony viability as a consequence of inbreeding, has been a great deterrent to successful closed breeding programmes.

In this paper we examine some consequences of the population genetics of sex determination in honey bees. We present genetic models and a computer model which examine the effects of random mating in closed breeding populations. This is a first attempt to develop the basis for closed population systems of breeding.

\section{MODELS AND RESUltS}

\section{(i) The distribution of brood viability}

If a queen $\left(X_{i} X_{j}\right)$ mates once and mates with a male having a sex allele identical to one of hers (say, $X_{i}$ ), she will produce diploid eggs in the 
proportions $0.50 X_{i} X_{j}$ and $0.50 X_{i} X_{i}$. Since the $X_{i} X_{i}$ homozygotes are lethal, the viability of her diploid brood will be 0.50 . If there are only two alleles in the population, all queens will experience the loss of 50 per cent of their brood. However, if a new allele, $\boldsymbol{X}_{k}$, is introduced into the population, all $\boldsymbol{X}_{i} \boldsymbol{X}_{j}$ queens mating with a male carrying this allele will have 100 per cent diploid brood viability with offspring genotypes $X_{i} X_{k}$ and $X_{j} X_{k}$ in equal proportions. These queens will leave more progeny in the next generation, and therefore this $\boldsymbol{X}_{\boldsymbol{k}}$ allele will increase in frequency. In this case (all homozygotes are lethal, all heterozygotes have the same fitness) we know that the central equilibrium (all alleles equally frequent) is globally stable (Kimura, 1956; Mandel, 1959).

Assume that there are $k$ alleles in the population, each at equal frequency. Then the probability, $p$, that a queen will mate with a male having an allele identical to one of hers is $p=2 / k$. Assuming random mating, the probability that a queen mating $n$ times will mate with $y$ males having alleles matching one of hers is given by the binomial:

$$
P(Y=y)=\left(\begin{array}{l}
n \\
y
\end{array}\right)\left(\frac{2}{k}\right)^{y}\left(1-\frac{2}{k}\right)^{n-y}
$$

in which $y=0,1,2, \ldots, n ; Y$ is a random variable of $y$.

If we assume that males of all matings contribute an equal amount of equally viable sperm, and assume equal sperm mixing, then the brood viability, $V$, for a queen mating $n$ times is:

$$
V=1-\frac{y}{2 n} \text {. }
$$

The expected brood viability in a population at allele frequency equilibrium is therefore given by:

$$
\begin{aligned}
E(V) & =\bar{V}=E\left(1-\frac{y}{2 n}\right) \\
& =1-\frac{1}{k}
\end{aligned}
$$

(see also Woyke, 1976).

The variance in brood viability is given by:

$$
\begin{aligned}
\sigma_{v}^{2} & =\operatorname{var}\left(1-\frac{y}{2 n}\right) \\
& =\frac{1}{2 n}\left(\frac{1}{k}\right)\left(1-\frac{2}{k}\right)
\end{aligned}
$$

(see also Adams et al., 1977).

Note that although the mean brood viability depends only on the number of alleles in the population, the variance in brood viability among queens is a function both of the number of matings and of the number of alleles in the population. Since the variance is inversely proportional to $n$, as the number of matings increases each queen is more likely to have her brood viability close to that of the population mean. 


\section{(ii) Effective population size and the number of alleles}

The mean brood viability $(\vec{V})$ of a population at equilibrium depends only on the number of sex determining alleles. The number of alleles that can be maintained in a random mating population at equilibrium is dependent upon:

(1) selection;

(2) rate of mutation and migration;

(3) the size of the breeding population $\left(N_{e}\right)$ (Wright, 1939, 1965; Kimura and Crow, 1964; Yokoyama and Nei, 1979).

Equation (26) from Kimura and Crow (1964) has been used to estimate the equilibrium number of alleles in populations of honey bees (Kerr, 1967; Cornuet, 1980). However, their model was developed for the case of symmetric overdominant selection in diploid organisms and was subsequently corrected by Yokoyama and Nei (1979) for the sex-linked male haploid case of honey bees. As expected, the Yokoyama and Nei model demonstrates analytically that as the effective breeding population size increases, more alleles can be maintained at mutation-selection-drift equilibrium.

The inbreeding effective population size $\left(N_{e}\right)$ in haplodiploid organisms is given by:

$$
N_{e}=\frac{9 N_{m} N_{f}}{4 N_{m}+2 N_{f}}
$$

in which

$$
\begin{aligned}
& N_{m}=\text { the number of breeding males } \\
& N_{f}=\text { the number of breeding females }
\end{aligned}
$$

(Wright, 1933).

Since honey bee males mate just once and die, we can alter the parameters of equation (1) in terms of $n$, the number of times each female mates, and $N_{f}$ (assuming equal contribution of sperm by males):

$$
N_{e}=\frac{9 n N_{f}}{4 n+2} \text {. }
$$

Note that $N_{e}$ increases linearly with number of females, but approaches a limit of $9 N_{f} / 4$ (see also Crozier 1979) very rapidly as the number of matings increases. The gain in $N_{e}$ is negligible for additional matings above $n=10$.

(iii) Rate of loss of alleles in finite populations: computer simulation

This simulation is designed to investigate the rate of loss of sex alleles and brood viability in artificial populations that are genetically isolated and maintained by instrumental insemination. Estimates of the number of sex alleles in "open" mating populations range from 6 to 18.9 (Mackensen, 1955; Laidlaw, et al., 1956; Kerr, 1967; Woyke, 1976; Adams et al., 1977). We start with an infinite number of males and females in a population with 12 alleles (a likely intermediate value). All alleles are at equal frequency and in Hardy-Weinberg proportions for females. A finite number of males and females are then selected at random as the first generation of a closed breeding population. 
This model simulates the conditions of:

(1) sex linkage;

(2) sex determination;

(3) random mating;

(4) finite population size;

for $\mathbf{4 0}$ generations. Mutation is not considered because of the short time period involved.

The sequence of events are:

(1) Select $N_{f}$ females at random.

(2) Select ten males at random from an infinite pool to mate with each female (the actual numerical sex ratio is several thousand males for each female; $n=10$ is a realistic estimate of natural conditions

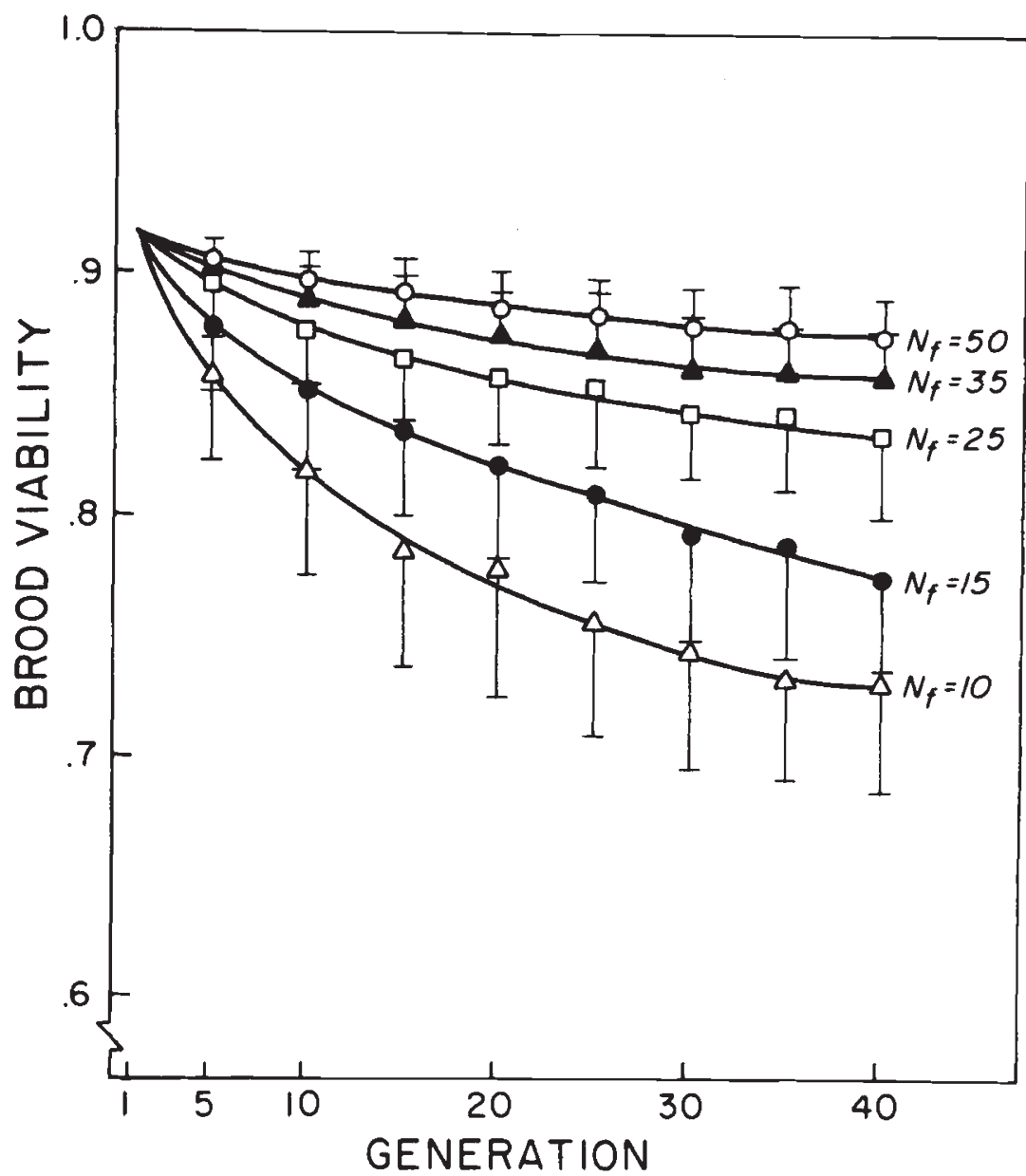

Fig. 1.-Results of the computer simulation. The means and standard deviations of brood viability over generations are plotted for different numbers of colonies $\left(N_{f}\right)$ maintained in closed populations. Each queen mated with 10 males; there were 12 alleles at equal frequency in generation one. Standard deviations are shown on one side only for the sake of clarity. 
(Page, 1980) and more matings result in little increase in $N_{e}$ (see equation 2)).

(3) Each female lays an infinite number of eggs, both diploid and haploid. New male (haploid) genotype frequencies depend only on female genotype frequencies; new female (diploid) genotype frequencies depend on the mating.

(4) All diploid homozygotes die; all heterozygotes are assumed to be equally fit.

Notice that drift occurs in each sex independently (see events 1 and 2 above). Since this will cause divergence in allele frequencies between the sexes, realized heterozygosity in females in the next generation will, on the average, be slightly larger than would be expected on the basis of the mean allele frequency in the population. (For autosomal loci, different

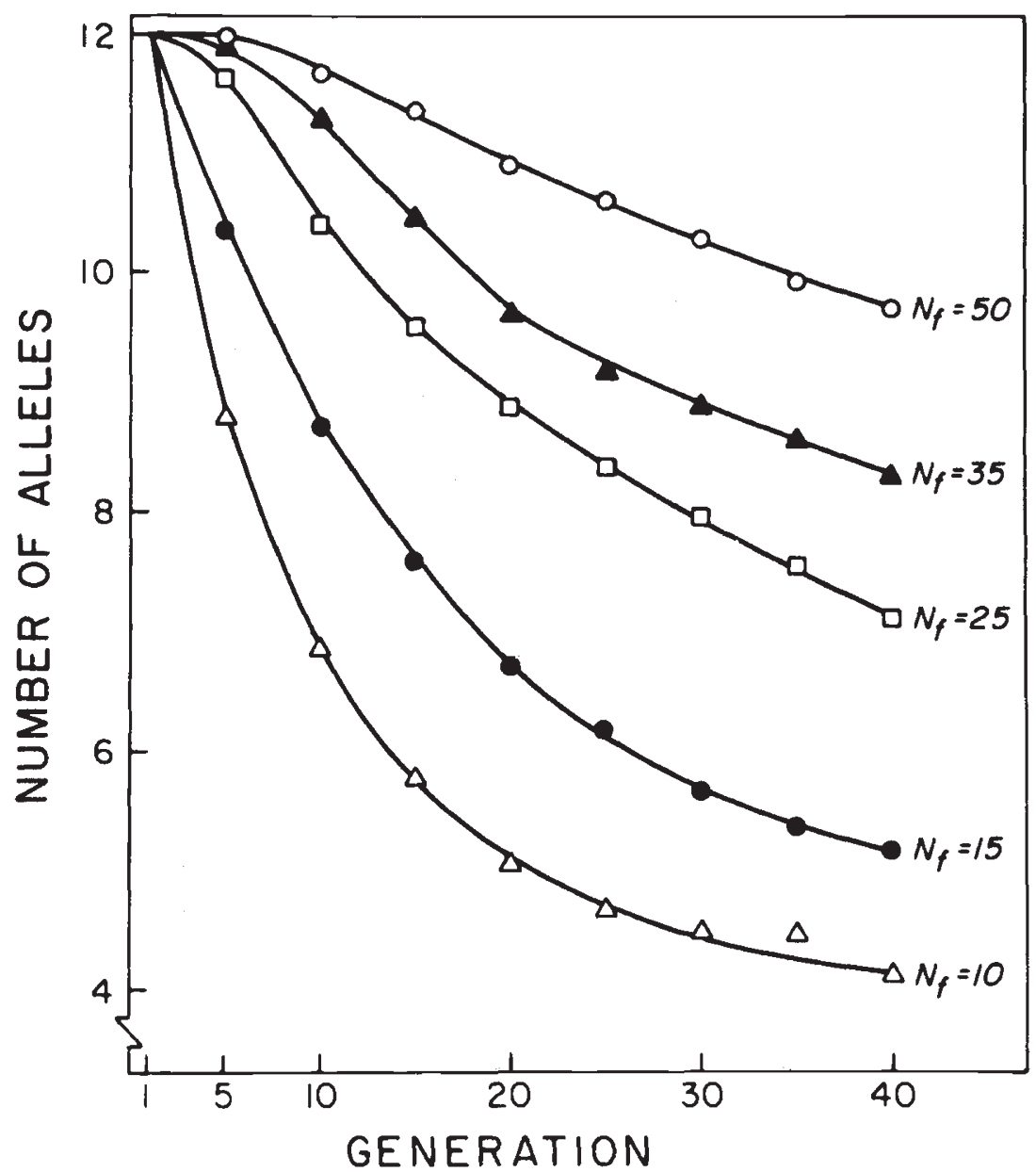

Fig. 2.-Results of the simulation. The expected numbers of alleles over generations for different numbers of colonies $\left(N_{f}\right)$ maintained in closed populations. Initial conditions are as in fig. 1. 
male and female allele frequencies always give heterozygote excess; this is not strictly true for sex-linked loci.)

All summary statistics are calculated just after step (3). One hundred iterations of generations 1 through 20 , and 50 iterations of generations 21 through 40 are used to calculate the means and standard deviations shown (figs. 1 and 2).

The decay with time of the brood viability and of the number of alleles maintained are shown in figs. 1 and 2 respectively. Even after 40 generations of drift, expected brood viabilities and numbers of alleles are well above the mutation-selection-drift equilibrium levels predicted by Yokoyama and Nei (1979). Also, the variance in brood viability decreases as population size increases, as expected. In fig. 3 are shown the realized probabilities that brood viabilities are at least 0.85 (a low acceptable value for commercial colonies) calculated from 50 iterations of the simulation for every generation.

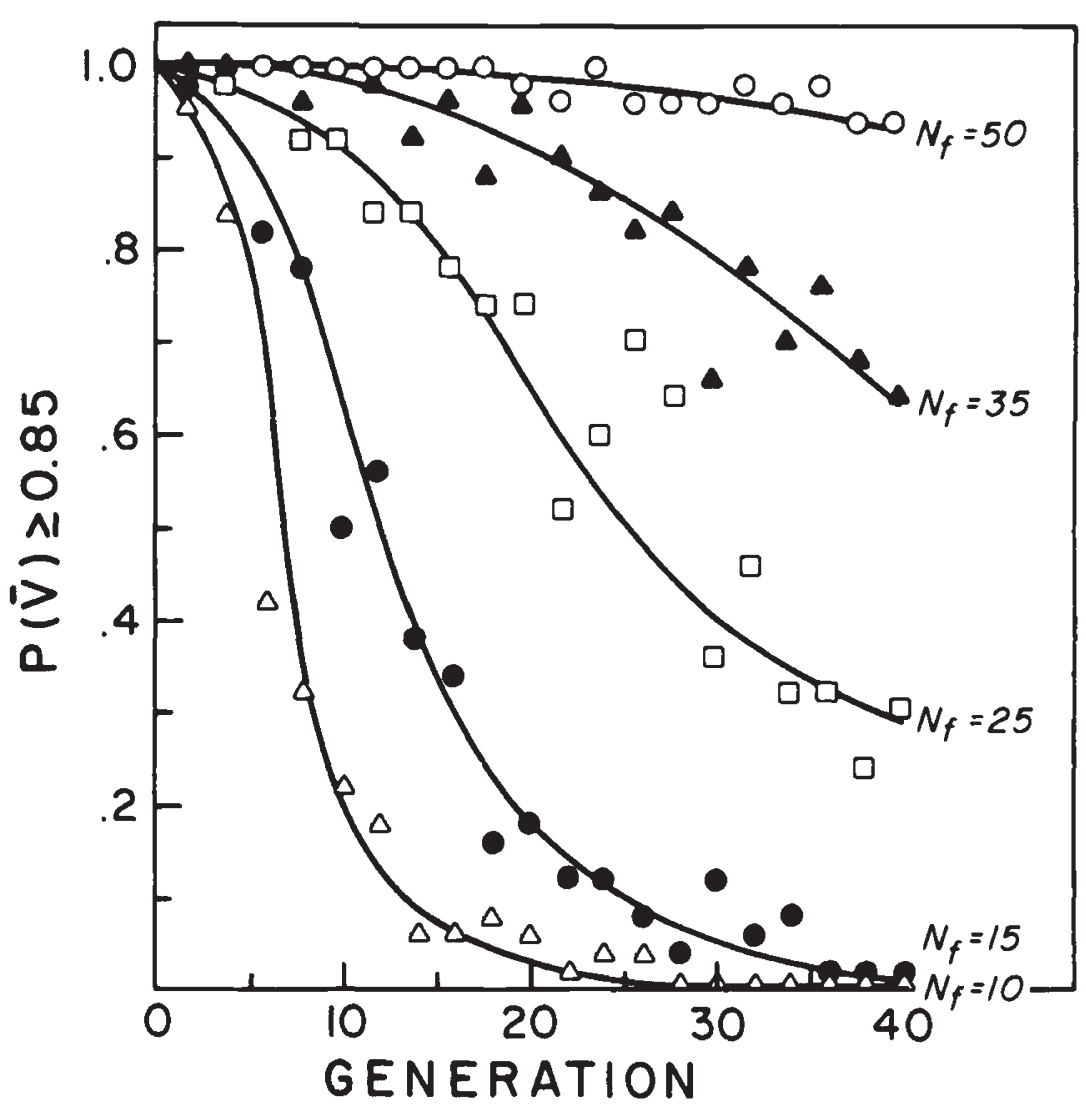

Frg. 3. The probability that a closed population of size $N_{f}$ will have an average brood viability of at least $\mathbf{0 \cdot 8 5}$, calculated for each of $\mathbf{4 0}$ generations (see fig. 2). Probabilities shown are the proportion of runs (out of 50 ) of the simulation in which actual brood viability was greater than 0.85 in the indicated generation. 


\section{Discussion}

The maintenance of closed populations of honey bees is important to selective breeding and stock maintenance programmes. Current practices of open queen (natural) mating result in virtual panmixia and hence reduced control over mass selection. Stock maintenance and controlled breeding programmes will become of paramount importance with the inevitable introduction of the "Africanized" genetic stock from South America into the major queen and package bee producing areas of the United States. Populations can be closed to introgression of "Africanized" alleles by instrumental insemination techniques. Once these populations are closed, loss of brood viability becomes a major consideration.

The results of this work show that a high average brood viability can be maintained over relatively long periods of time ( 1 generation $=1$ year in temperate zones). With a minimum of 50 colonies $\left(N_{f}=50\right)$, little loss of viability is expected over 40 generations. In fact, the probability is 0.95 that the mean viability will be at least 85 per cent after this period.

Variance in queen performance, as a consequence of sex determination, can be controlled by regulating the number of matings. Virtually every queen produced will have the same mean brood viability if a large number of males contribute sperm to each queen. Methods now exist whereby sperm from many males (hundreds or even thousands) can be collected and mixed in one lot, then used to inseminate many queens (Kaftangolu and Peng, 1980a, $b$ ).

Acknowledgements.-Our thanks go to Dr James F. Crow for his helpful comments on the manuscript. We are especially grateful to Dr Harry $\mathrm{H}$. Laidlaw, Jr. for supporting this work. This project was funded primarily by the Bee Genetics programme of the Agricultural Development Systems Project, Arab Republic of Egypt, University of California, United States Agency for International Development, H. H. Laidlaw, principal investigator. Computer simulations were funded in part by a grant to Dr R. C. Lewontin, Department of Energy Contract \# DE-AC02-76EV02472.

\section{REFERENCES}

ADAMS, J., ROTHMAN, E. D., KERR, W. E., AND PAULINO, Z. L. 1977. Estimation of the number of sex alleles and queen matings from diploid male frequencies in a population of Apis mellifera. Genetics, 86, 583-596.

CORNUET, J. M. 1980. Rapid estimation of the number of sex alleles in panmictic honey bee populations. J. Apic. Res., 19, 3-5.

CROZIER, R. H. 1979. Genetics of sociality. In: H. R. Herman (ed.) Social Insects Vol. I, Academic Press, pp. 223-286.

KAFTANOGLU, O., AND PENG, Y.-S. 1980a. A new syringe for semen storage and instrumental insemination of queen honey bees. J. Apic. Res., 19, 73-76.

KAFTANOGLU, O., AND PENG. Y.-S. $1980 \mathrm{~b}$. A washing technique for collection of honey bee semen. J. Apic. Res., 19, 205-211.

KERR, W. E. 1967. Multiple alleles and genetic load in bees. J. Apic. Res., 6, 61-64.

KIMURA, M. 1956. Rules for testing the stability of a selective polymorphism. Proc. Natl. Acad. Sci. U.S., 42, 336-340.

KIMURA, M., AND CROW, J. F. 1964. The number of alleles that can be maintained in a finite population. Genetics, 49, 725-738.

LAIDLAW, H. H., JR., GOMES, F. P. AND KERR, W. E. 1956. Estimations of the number of lethal alleles in a panmictic population of Apis mellifera. Genetics, 41, 179-188.

MACKENSEN, O. 1951. Viability and sex determination in the honey bee. Genetics, 36 , 500-509. 
MACKENSEN, O. 1955. Further studies on a lethal series in the honey bee. J. Heredity, 46, $72-74$.

MANDEL, S. P. H. 1959. The stability of a multiple allelic system. Heredity, 13, 289-302.

PAGE. R. E. 1980. The evolution of multiple mating behavior by honey bee queens (Apis mellifera L.). Genetics, 96, 263-273.

WOYKE, J. 1963. What happens to diploid drone larvae in a honey bee colony. J. Apic. Res., $2,73-75$.

WOYKE, J. 1976. Population genetic studies on sex alleles in the honey bee using the example of the Kangaroo Island bee sanctuary. J. Apic. Res., 15, 105-123.

WRIGHT, S. 1933. Inbreeding and homozygosis. Proc. Natl. Acad. Sci. U.S., 19, 411-420.

WRIGHT, S. 1939. The distribution of self-sterility alleles in populations. Genetics, 24, $538-553$.

WRIGHT, S. 1965. The distribution of self-incompatibility alleles in populations. Evolution, $18,609-619$.

YOKOYAMA, S., AND NEI, M. 1979. Population dynamics of sex-determining alleles in honey bees and self-incompatibility alleles in plants. Genetics, 91, 609-626. 